
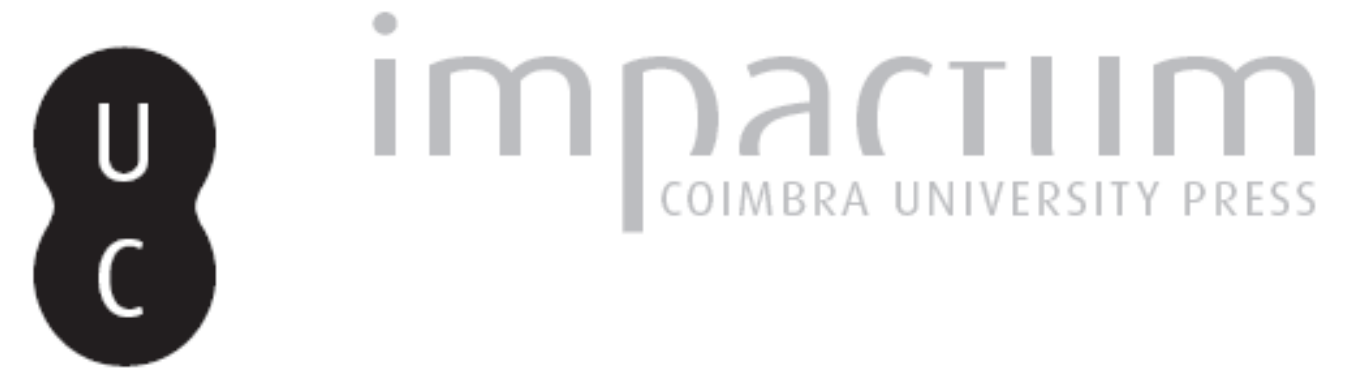

\title{
La psichicità dell'anima del mondo e il divenire precosmico
}

\section{Autor(es): $\quad$ Ferrari, Franco}

Publicado por: International Plutarch Society

URL persistente:

URl:http://hdl.handle.net/10316.2/36361

DOI:

DOI:http://dx.doi.org/10.14195/0258-655X_9_2

Accessed : $\quad$ 26-Apr-2023 14:31:40

A navegação consulta e descarregamento dos títulos inseridos nas Bibliotecas Digitais UC Digitalis, UC Pombalina e UC Impactum, pressupõem a aceitação plena e sem reservas dos Termos e Condições de Uso destas Bibliotecas Digitais, disponíveis em https://digitalis.uc.pt/pt-pt/termos.

Conforme exposto nos referidos Termos e Condições de Uso, o descarregamento de títulos de acesso restrito requer uma licença válida de autorização devendo o utilizador aceder ao(s) documento(s) a partir de um endereço de IP da instituição detentora da supramencionada licença.

Ao utilizador é apenas permitido o descarregamento para uso pessoal, pelo que o emprego do(s) título(s) descarregado(s) para outro fim, designadamente comercial, carece de autorização do respetivo autor ou editor da obra.

Na medida em que todas as obras da UC Digitalis se encontram protegidas pelo Código do Direito de Autor e Direitos Conexos e demais legislação aplicável, toda a cópia, parcial ou total, deste documento, nos casos em que é legalmente admitida, deverá conter ou fazer-se acompanhar por este aviso.

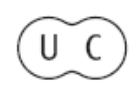


Ploutarchos, n.s.

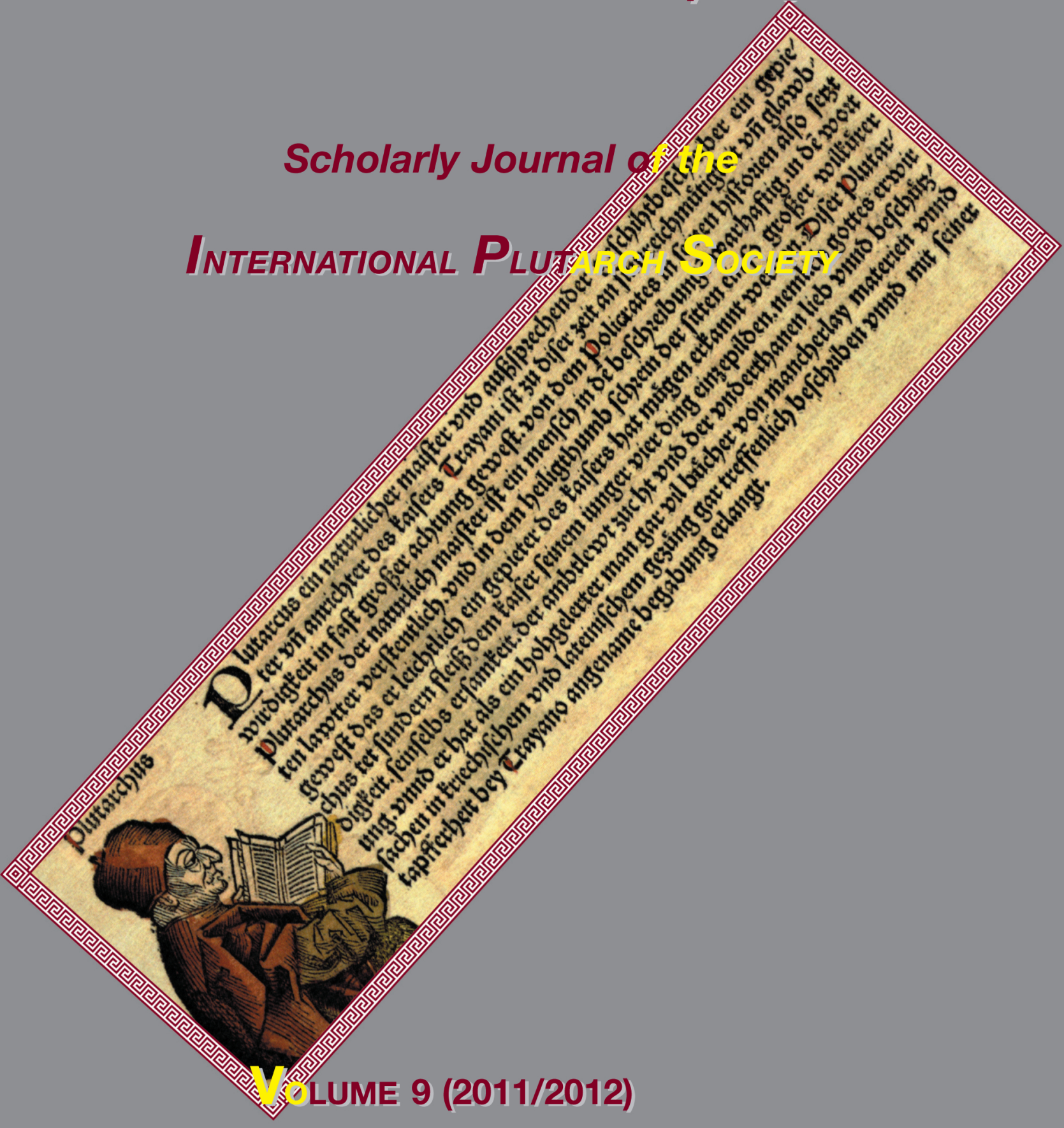

University of Málaga (Spain) Utah State University, Logan, Utah (U.S.A.) 


\title{
La psichicità dell'anima del mondo e il divenire precosmico secondo Plutarco* da \\ Franco Ferrari \\ Università di Salerno \\ fr.ferrari@unisa.it
}

\begin{abstract}
Plutarch's treatise On the Generation of the Soul in Plato's Timaeus can be considered as the most important philosophical work in the Moralia. It is dedicated to the interpretation of Timaeus' passage on the ontological generation and numerical division of the world-soul (35A ss.). According to the first interpreters (Xenocrates and Crantor), in order to understand the significance of Plato's words, it is very important to clarify the meaning of the two fundamental ingredients of the world-soul, i.e. the indivisible and ever invariable being and the divisible being that comes to pass in the case of bodies. In the first section of his work Plutarch critizises the interpretations proposed by Xenocrates and Crantor because they are not capable to indicate among the components mentioned by Plato the psychic ingredient of the world-soul. According to Plutarch this psychic element must be identified with the divisible being, which is a reference to a precosmic soul. This irrational soul moves the precosmic body (matter) from eternity. It is the same soul mentioned by Plato in the 10 book of the Laws. The precosmic soul, as the soul itself, is the cause of chaotic movement of the matter and also the principle of evil. This irrational soul is responsable for all psychic elements present in the world-soul, i.e. the kinetikon, the phantastikon, and the kritikon. The generation of the world-soul consists in the fact that the precosmic irrational soul receives a partecipation in the intelligible, i.e. in the indivisible being, so that it becomes ordered. The hypothesis of this precosmic soul as substrat of the worldsoul allow to Plutarch to solve a set of exegetical problems in Plato's text.
\end{abstract}

Key-Words: Moralia. Plato. Timaeus. Plutarch's philosophical thought. World-soul. Precosmic soul.

* Questo saggio nasce da una serie di lezioni che ho tenuto presso l'"École doctoral de Philosophie antique et médiévale" su invito dalla "Conférence universitaire de Suisse occidentale” sul tema Les exégèses du 'Timée' de Platon (24-26 novembre 2011). Ringrazio Filip Karfik e Tiziana Suarez (Università di Fribourg) per l'invito e la squisita ospitalità. 
I

uando, probabilmente
a cavallo tra gli anni
90 del I e l'inizio del II
secolo d.C. ${ }^{1}$, Plutarco scrisse il trattato De animae procreatione in 'Timaeo', che costituisce forse la sua opera filosofica più impegnativa, almeno tra quelle a noi pervenute, sulla sezione del Timeo in cui si trova la descrizione della genesi ontologica e della divisione numerica dell'anima del mondo esisteva una tradizione esegetica ormai molto lunga, della quale lo stesso Plutarco rappresenta un testimone prezioso. Egli menziona e discute le interpretazioni dei primi accademici, ossia Senocrate e Crantore, ma anche le posizioni di autori come Teodoro di Soli, contemporaneo di Crantore, l'aristotelico Clearco di Soli e soprattutto Posidonio di Apamea, sebbene si possa presumere che non disponesse direttamente degli scritti di tutti questi esegeti ma si servisse in taluni casi come fonte di Eudoro di Alessandria, il quale aveva composto un importante commento (vं ó $\mu v \eta \mu \alpha$ ) al Timeo, che Plutarco conosceva molto bene e dal quale attinse con una certa sistematicità ${ }^{2}$.
È difficile stabilire se ai tempi di Plutarco esistesse un'esegesi canonica della concezione platonica dell'anima del mondo. Ciò che Plutarco afferma all'inizio dell'opera, in particolare nei primi quattro capitoli, induce comunque a ritenere che almeno su un punto la maggioranza dei platonici fosse concorde, vale a dire nell'attribuire un significato metaforico alle parole contenute nel Timeo sulla generazione del mondo e della sua anima. Una delle ragioni per cui Plutarco decise di comporre uno scritto dedicato alla concezione platonica dell'anima del mondo risiede esattamente nell'esigenza di opporsi a questa communis opinio (An. procr. 1. 1012B), affermando la natura letterale della cosmogenesi del Timeo, la quale si riferisce a un evento reale, rappresentato, come vedremo, dall'azione di ordinamento che il demiurgo opera nei confronti di un sostrato indeterminato ${ }^{3}$.

In ogni caso l'esistenza di un certo accordo tra i platonici circa il carattere metaforico della descrizione della genesi dell'anima e del cosmo non deve indurre a ritenere che nel I secolo

1 Rimane questo l'arco temporale nel quale va verosimilmente collocata la stesura di questo trattato: cfr. in proposito CHERNISS, 1976, pp. 133-34, HERSHBELL, 1987, pp. 237-38 e FERRARI, 2002, p. 11 e p. 215 n. 1.

2 In realtà la questione se Plutarco avesse accesso diretto alle opere di Senocrate e Crantore oppure ne conoscesse le dottrine solo di seconda mano (via Eudoro o addirittura Posidonio) rimane aperta. Si veda il quadro delle posizioni della critica fornito da HershBell, 1987, p. 239.

3 Sull'interpretazione letterale plutarchea della genesi dell'universo descritta nel Timeo cfr. BALtes, 1976, pp. 38-45. 
d.C. il passo del Timeo relativo alla composizione e alla divisione dell'anima ammettesse un'interpretazione univoca. L'accordo tra gli esegeti di Platone si limitava probabilmente all'accettazione dell'interpretazione didascalica, che sembra avere ormai preso il sopravvento nei confronti dell'esegesi letterale, proposta da Aristotele e poi diffusa nel periodo ellenistico, soprattutto tra i rivali di Platone e in particolare tra gli epicurei. Per quanto concerne invece l'interpretazione della struttura compositiva dell'anima cosmica le opinioni dei platonici divergevano in maniera piuttosto significativa.

Plutarco individua due principali linee esegetiche, quella aritmeticomatematica, che risaliva a Senocrate (il quale identificava l'anima con il numero), e quella epistemologica, il cui massimo esponente fu Crantore di Soli, il quale vedeva nell'anima una mescolanza di tutti gli elementi che essa ha il compito di conoscere ( $A n$. procr. 1-2. 1012D-1013A). E non si può escludere che altri punti di vista circolassero in questo periodo. Del resto, la situazione del platonismo nel I secolo d.C. era estremamente fluida, e il tramonto dello scetticismo accademico, che per circa due secoli aveva egemonizzato la vita della scuola fondata da Platone, aveva determinato un proliferare di posizioni differenti, che solo a partire dal IV secolo si sarebbero unificate in qualcosa di simile a una nuova ortodossia.

\section{II}

Come è noto, lo scritto di Plutarco è diviso in due parti chiaramente distinte, ciascuna delle quali viene introdotta dalla citazione delle linee del Timeo di cui viene fornita l'esegesi ${ }^{4}$. La prima parte del trattato, che è di gran lunga la più estesa, occupa $\mathrm{i}$ capp. 1-27 (1012B-1027A) e si concentra sulle linee 35A1-B4 del Timeo, dove si trova la descrizione della composizione dell'anima cosmica ad opera del demiurgo, mentre la seconda è contenuta nei capp. 29-33 (1027A-1033C $)^{5}$ e affronta l'esegesi delle linee 35B436B5 dove viene introdotta la celebre e misteriosa sequenza numerica della

4

Il testo riportato da Plutarco si discosta in alcuni punti da quello dei manoscritti platonici. Altrove ho cercato di dimostrare che le variazioni introdotte da Plutarco sono funzionali a supportare l'esegesi che egli si appresta ad avanzare: cfr. FERRARI, 1999, pp. 332-38. Si vedano in proposito anche le acute osservazioni di OPSOMER, 2004, pp. 139-42 e 159-61.

5

Come è noto, la numerazione dei capitoli è resa problematica da uno spostamento intervenuto nella tradizione manoscritta di una sezione di testo (capp. 21-30) dalla sua collocazione naturale (dopo il cap. 11). Anche dopo che ci si rese conto dell'inversione, si è conservata la numerazione della tradizione manoscritta, la quale non riproduce in modo corretto l'ordine del testo: cfr. Cherniss, 1976, p, 134, Hershbell, 1987, p. 238 n. 23 e p. 243, e FerRari, 2002, p.10 n. 9. 
divisio animae, ossia la serie dei numeri in base ai quali il demiurgo avrebbe diviso la massa psichica precedentemente formata ${ }^{6}$.

Entrambe le parti dell'opera di Plutarco presentano numerosi motivi di interesse, e la svalutazione di cui è stata fatta oggetto la seconda, considerata filosoficamente irrilevante (e non tradotta, per esempio, da Pierre Thévenaz all'inizio della sua monografia sul trattato $^{7}$ ), appare del tutto ingiustificata, come ho cercato di dimostrare in numerosi scritti che qui non è possibile menzionare ${ }^{8}$. Non c'è dubbio, comunque, che la sezione dedicata alla composizione ontologica dell'anima non sia solo la più estesa ma anche quella filosoficamente più interessante. Di essa, e in particolare di ciò che Plutarco riconosce essere l'aspetto

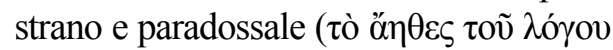

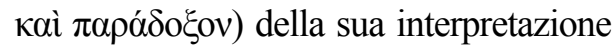
(An. procr. 5. 1014A), intendo occuparmi nelle pagine che seguono.

Prima di entrare nel vivo dell'esegesi di Plutarco è opportuno riportare la tra- duzione del testo del Timeo intorno al quale verte la prima parte del trattato plutarcheo. Come è noto, dopo avere descritto la composizione del corpo del mondo, Timeo delinea le operazioni che il demiurgo ha messo in atto per "generare" l'anima cosmica. Egli afferma:

Tra l'essere indivisibile e sempre allo stesso modo e l'essere divisibile che diviene nei corpi, mischiò nel mezzo un terzo genere di essere composto da entrambi; poi, per quel che riguarda la natura dell'identico e quella del diverso, proprio allo stesso modo compose insieme un terzo genere nel mezzo della loro specie indivisibile e di quella divisibile nei corpi. E prendendo questi tre ingredienti li mescolò tutti in una sola forma, armonizzando con la forza la natura del diverso, che è refrattaria alla mescolanza, con l'identico, e li mescolò insieme all'essere, e fece di tre un'unica realtà (Tim. 35A1-B1).

$\mathrm{Si}$ tratta di un testo complesso, che ha dato origine a una quantità impressionante di interpretazioni, sia

6 Sulla natura esegetica dello scritto plutarcheo, che può venire considerato a tutti gli effetti un "commentario" (e per la precisione un "commentario specialistico"), cfr. FERRARI, 2002, pp. 7-21 e Opsomer, 2004, p. 139. Un quadro di insieme, informato e prudente, delle interpretazioni antiche e moderne del passo del Timeo sull'anima del mondo viene fornito da BRISSON, 1994, pp. 267-354.

7 Molto esplicite le parole con cui THÉVENAz, 1938, p. 10 giustifica la sua scelta di tralasciare la traduzione e la discussione della seconda parte del trattato: "les développements mathématiques, arithmologiques et musicaux qui doivent étayer l'interprétation de la division harmonique de l'âme, n'entrent pas dans le cadre de mon étude, qui s'en tient aux vues de métaphysique générale".

8 Rinvio per comodità al mio commento alla seconda parte dello scritto: FERRARI, 2002. 
nell'antichità sia tra i commentatori moderni. Non essendo qui possibile dare conto delle diverse opzioni esegetiche, mi limiterò a riassumere l'interpretazione che mi pare la più ragionevole e che può vantare illustri sostenitori tra i platonisti dell'ultimo secolo.

Platone descrive nel testo appena citato due livelli di mescolanza: la prima comporta l'unione di una parte indivisibile e di una divisibile e interessa tre determinazioni, vale a dire l'essere, l'identico e il diverso: l'essere

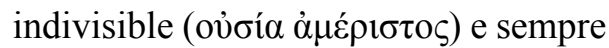
identico a sé viene mescolato con l'essere divisibile che diviene nei corpi

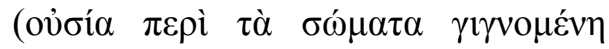
$\mu \varepsilon \rho ı \tau \tau \dot{)}$, dando origine a un essere mediano ('́v $\mu \varepsilon ́ \sigma \omega)$; allo stesso modo l'identico indivisibile viene unito all' identico divisibile per formare un identico intermedio, e il diverso indivisibile viene mescolato con il diverso divisibile per dare origine a un diverso mediano. Successivamente il demiurgo interviene sulle tre essenze prodotte dalla prima mescolanza generando una mescolanza finale, formata dall'essere, dall'identico e dal diverso mediani; egli unisce questi tre ingredienti e forma in questo modo un'unica realtà, cioè la massa psichica, sulla quale opererà le divisioni numeriche descritte nella seconda parte del passo ${ }^{9}$.

Per mezzo di questo complesso schema compositivo Platone sembra voler sottolineare la collocazione intermedia dell'anima del mondo, la quale risulta formata nella sua mescolanza finale da tre realtà intermedie: l'essere, l'identico e il diverso. Questa intermedietà dovrebbe consentirle di svolgere una funzione mediatrice tra l'ambito delle idee e quello dei fenomeni spazio-temporali. E poi probabile che la presenza tra gli ingredienti dell'anima cosmica di essere, identico e diverso sia da mettere in rapporto alla discussione dei "generi sommi" contenuta nel Sofista, sebbene la natura della relazione tra $\mathrm{i}$ due dialoghi continui a essere motivo di innumerevoli contrasti tra gli studio$\mathrm{si}^{10}$. Fin qui dunque Platone.

\section{III}

Se si presta fede alla ricostruzione delle concezioni circolanti nell'Accademia proposta da Plutarco, sembra inevitabile concludere che le prime interpretazioni del passo platonico intorno alla genesi dell'anima del mondo ruotassero intorno al problema di stabilire quali fossero esattamente gli ingredienti menzionati nel testo, vale a dire che cosa intendesse Platone con le espressioni

9 Questa interpretazione, che può considerarsi ormai classica, viene esposta in maniera molto chiara da BrISSON, 1994, pp. 270-75; si veda anche FERRARI, 1999, pp. 328-32.

10 Sul tentativo plutarcheo di integrare la trattazione dei "generi sommi" del Sofista nell'ambito della cosmologia e dell'ontologia di Platone cfr. Schорте, 1994, pp. 75-138. 
"essere indivisibile" ed "essere divisibile nei corpi". Del resto anche per Plutarco una corretta interpretazione della dottrina platonica dell'anima richiede innanzitutto il chiarimento degli ingredienti a partire dai quali questa entità viene costruita dal demiurgo.

La posizione personale di Plutarco inizia a profilarsi proprio nelle prime pagine dello scritto, quando egli polemizza con le interpretazioni di Senocrate e Crantore. Ad entrambi si può addebitare la responsabilità di non avere colto la specificità dell'anima, cioè di non avere individuato tra gli ingredienti menzionati da Platone l'elemento psichico, vale a dire ciò che è responsabile delle caratteristiche proprie dell'anima, che sono, per Plutarco, la ricettività ( $\tau$ ò $\pi \alpha \theta \eta \tau$ «óv), ossia la capacità di accogliere impressioni, la motricità ( $\tau$ ò

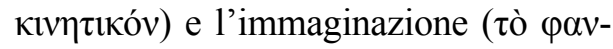
$\tau \alpha \sigma \tau$ «óv), cioè la facoltà di produrre immagini (An. procr. 23. 1023D) ${ }^{11}$. Né Senocrate né Crantore si sono dimostrati capaci di isolare nel passo platonico l'ingrediente in virtù del quale l'anima del mondo è un'anima.

Per Senocrate, come è noto, l'essenza dell'anima consiste nell'essere nu- mero, e per la precisione "numero

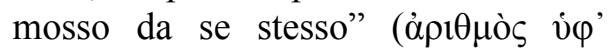

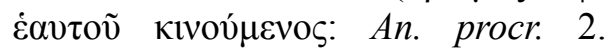
1012D). Trattandosi di un numero, i suoi componenti fondamentali sono l'uno, che corrisponde all'essere indivisibile, e la molteplicità, che corrisponde all'essere divisibile nei corpi. L'aggiunta di identico e diverso, i quali rappresentano rispettivamente $\mathrm{i}$ principi della permanenza e del movimento, segna la genesi dell'anima,

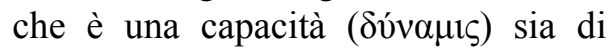
stabilizzare che di muovere (1012EF). Secondo Plutarco nessuno di questi ingredienti è in grado di spiegare la natura dell'anima, perché nessuno sembra responsabile della psichicità, cioè della presenza in essa dell'insieme dei caratteri che qualificano l'anima in quanto anima. In particolare l'errore di Senocrate consiste nella confusione tra

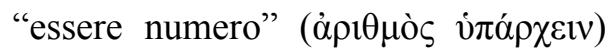
ed "essere costituito in base al numero"

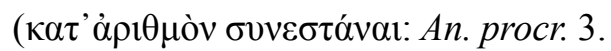
1013D): l'anima è certamente costituita in base a rapporti numerici, come risulta evidente dal passo della divisio animae, dal momento che essa è divisa sulla base di rapporti aritmetici, geometrici e armonici; ma ciò non

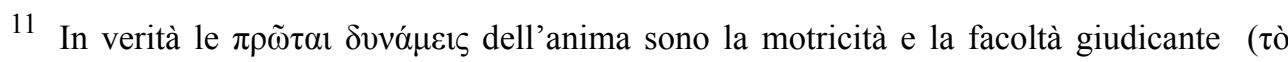

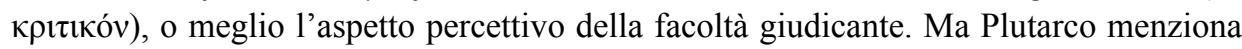

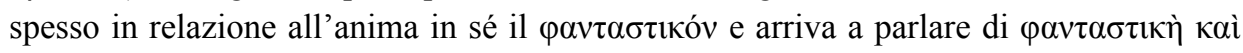

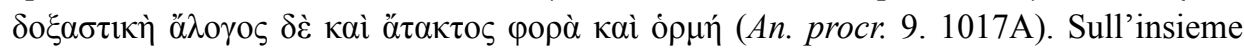
delle caratteristiche che definiscono la specificità dell'anima, cioè dell'elemento psichico in se stesso, sono fondamentali le riflessioni di Deuse, 1983, pp. 16-20; si veda anche BAltes, 2000, pp. 248-50. 
significa che essa sia un numero ${ }^{12}$. In

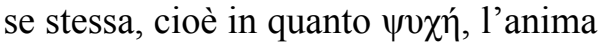
è diversa dal numero e la presenza del numero ha la funzione di rendere la sostanza psichica adatta allo scopo per cui è stata generata, che consiste nell'ordinare i processi che pervadono il cosmo sensibile.

Neppure l'esegesi epistemologica di Crantore risulta, secondo Plutarco, soddisfacente. Per Crantore dietro all'essere indivisibile e all'essere divisibile nei corpi di cui parla Platone si nascondono rispettivamente la na-

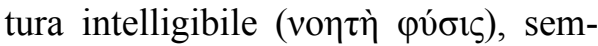
pre identica a sé, e quella ricettiva e

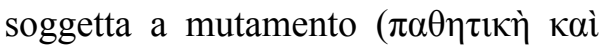
$\mu \varepsilon \tau \alpha \beta \lambda \eta \tau \eta ́)$, alle quali si aggiungono la natura dell'identico e quella del diverso (An. procr. 2. 1012F-1013A). Dal momento che, secondo Crantore, la funzione dell'anima consiste nel conoscere la realtà individuando differenze e somiglianze in ciascun ambito, e dal momento che gli ambiti ammessi da Platone sono due, vale a dire la sfera intelligibile e quella sensibile, l'anima è formata da quattro ingredienti: l'essere intelligibile, quello sensibile, l'identico e il diverso, perché solo così, ossia solo se composta dagli stessi principì di cui è formata la realtà, essa può arrivare a conoscere quest'ultima, sulla base della norma secondo la quale "il simile viene conosciuto dal simile".

Così facendo, tuttavia, anche Crantore si dimostra incapace di individuare la componente psichica che entra nella costituzione dell'anima cosmica. Infatti i quattro ingredienti da lui menzionati contribuiscono a formare qualsiasi realtà e nessuno di essi fornisce ciò che rende l'anima cosmica un'anima. In altre parole, la genesi dell'anima descritta da Crantore non si distingue dalla genesi di qualsiasi altra realtà, e finisce per lasciare senza spiegazione ciò che fa dell'anima cosmica un'anima ( $A n$. procr. 3. 1013B-C).

In realtà anche agli interpreti più recenti si possono muovere, secondo Plutarco, obiezioni simili. In effetti neppure Posidonio è immune dalla critica di non avere isolato la componente psichica all'interno della mescolanza dell'anima del mondo.

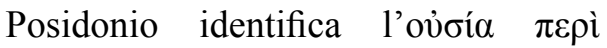

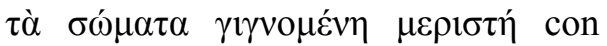
l'essenza dei limiti ( $\tau \tilde{\omega} v \pi \varepsilon \rho \alpha ́ \tau \omega v$ ov̉oía), cioè con principi di natura geometrica (22. 1023B). Quindi concepisce l'anima come "forma di ciò che

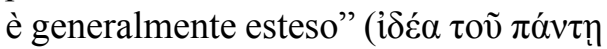
$\delta 1 \alpha \sigma \tau \alpha \tau o \tilde{)})$, finendo quindi per fare di essa una realtà geometrico-matematica, in maniera non dissimile da Senocrate e soprattutto da Speusippo ${ }^{13}$. Agli oc-

12 Sulle obiezioni che Plutarco rivolge a Senocrate continuano a sembrarmi utili le considerazioni di ThÉvenAz, 1938, pp. 57-69.

13 Per un esame approfondito della definizione di Posidonio e delle esegesi geometrico- 
chi di Plutarco, se è tuttosommato accettabile l'identificazione posidoniana dell'essere indivisibile con il

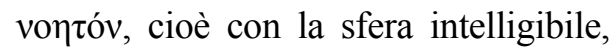
risulta assolutamente inconsistente l'assimilazione dell'essere divisibile nei corpi con la molteplicità, con i limiti o con qualsiasi altro principio di natura geometrico-matematica. Le sue parole non potrebbero essere più chiare:

Mentre è la partecipazione al principio intelligibile ad avere prodotto in essa [nell'anima del mondo] intelligenza e facoltà ra-

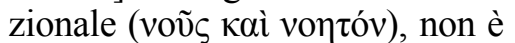
invece pensabile che opinioni e credenze, l'immaginazione e la impressionabilità ad opera delle

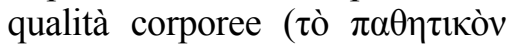

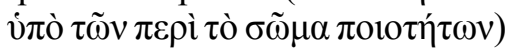
sopraggiungano nell'anima semplicemente da monadi, linee e superfici (23. 1023D).

Da questo passo si evince che secondo Plutarco l'esegesi della concezione platonica della genesi dell' anima del mondo ruoti intorno all'inter-

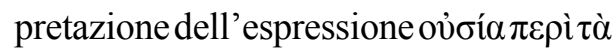

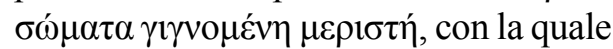
Platone designa uno degli ingredienti fondamentali dell'anima cosmica. Se infatti gli interpreti sembrano, con rare eccezioni (per es. Senocrate), disposti a riconoscere nell'essere indivisibile un riferimento al mondo delle idee o comunque alla sfera intelligibile e noetica, a proposito dell'essere divisibile nei corpi le posizioni sono diversificate e la tendenza a identificare questa entità con un'essenza di carattere geometricomatematico (Senocrate e Posidonio) o addirittura corporeo (Crantore), sembra a Plutarco assolutamente inaccettabile perché preclude la possibilità di individuare la componente psichica presente nell'anima del mondo.

\section{IV}

Per Plutarco, dunque, nessuno degli interpreti che lo hanno preceduto si è dimostrato in grado di individuare tra gli ingredienti di cui parla Platone quello che garantische la "psichicità" dell'anima cosmica. In altre parole, nessun ha isolato l'elemento psichico cheentranella composizionedell'anima del mondo e che è responsabile del fatto che quest'ultima sia un'anima. Il senso della strategia plutarchea viene restituito in maniera molto chiara da ciò che si legge nel capitolo 23:

È un'impresa, poi, dire da dove l'anima ha preso questo movimento di natura opinativa che percepisce il sensibile e che è diverso da quello noetico che culmina nella conoscenza, se non si ammette con fermezza che in questo passo non è l'anima in senso assoluto bensì l'anima del

matematizzanti dell'anima del mondo rinvio a FERRARI, 2002, pp. 277-83, con gli opportuni riferimenti alla ricca letterarura secondaria. Vale sempre la pena consultare THÉVENAZ, 1938, pp. 63-7. 
mondo che Platone costituisce a partire da entità preesistenti, cioè dall'essere migliore e indivisibile e da quello peggiore, che ha chiamato divisibile nei corpi ( $\pi \varepsilon \rho \grave{~} \tau \grave{\alpha}$ $\sigma \omega ́ \mu \alpha \tau \alpha \mu \varepsilon \rho \iota \sigma \tau \eta ́)$, il quale non è altro che il movimento di natura opinativa e rappresentativa congenere al sensibile (An. procr. 23. 1023F-1024A).

Plutarco rivendica a se stesso il merito, primo fra tutti i platonici, di avere isolato questa componente. $\mathrm{Si}$ tratta appunto di ciò che Timeo chiama

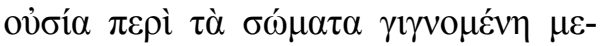

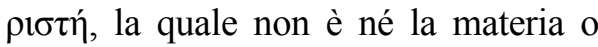
il corpo, né la molteplicità e neppure l'essenza dei limiti, bensì l'anima in sé

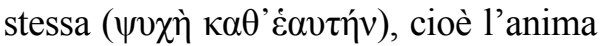
nella sua forma più pura e assoluta $(\dot{\alpha} \pi \lambda \tilde{\omega} \varsigma)$, e per l'esattezza l'anima precosmica irrazionale, la quale è eterna come la materia, cioè la corporeità priva di forma, e insieme alla materia rappresenta tutto ciò che esiste prima dell'intervento ordinatore del demiurgo.

Plutarco arriva a formulare l'ipotesi, per certi aspetti rivoluzionaria, dell'anima precosmica sulla base di un'analisi approfondita e di un'interpretazione letterale di ciò che Platone afferma a proposito della condizione dell'universo prima dell'intervento del demiurgo. Questa condizione presenta due aspetti, che sono inseparabili dal punto di vista "temporale" ma risultano distinguibili sul piano strettamente logico: si tratta dell'aspetto cinetico, rappresentato appunto dall'anima, e di quello corporeo, di cui è responsabile la materia (v๊ $\lambda \eta)$. Quando Platone afferma, ad esempio in Timeo, 30A, che dio "avendo così preso in mano tutto quanto era visibile, e che non era in riposo ma si muoveva in modo caotico e privo di ordine $(\pi \lambda \eta \mu \mu \varepsilon \lambda \tilde{\omega} \varsigma$ à $\tau \dot{\alpha} \kappa \tau \omega \varsigma$ ), lo condusse dal disordine all'ordine, ritenendo che questo fosse in tutto migliore di quello", egli intende, secondo Plutarco, sostenere che l'universo presenta nella sua fase precosmica due aspetti: uno corporeo, che determina la sua visibilità, e uno cinetico, che causa il movimento del corpo precosmico. Dal momento che il movimento di cui qui si parla è precedente l'azione ordinatrice del demiurgo, non può che trattarsi di un movimento disordinato e privo di regola perché in esso manca un principio intelligente e razionale.

Come si è anticipato all'inizio di questo saggio, secondo Plutarco l'atto di cosmogenesi costituisce un evento reale e non una condizione ontologica perenne (creatio perpetua), come invece ritenevano Senocrate, Crantore ed Eudoro. Ciò significa che gli accenni platonici a uno stato precosmico dell' universo si riferiscono a una condizione reale nella quale il tutto (cò $\pi \tilde{\alpha} v)$ si trovava prima dell'intervento dell'intelligenza demiurgica. Questo non significa che l'universo è stato generato nel tempo, perché, a rigore, il tempo nasce insieme all'universo, come Platone afferma in Tim. 38B. Significa 
però che esisteva una condizione dell'universo precedente rispetto a quella attuale e che l'universo è stato formato a partire da alcune entità preesistenti (la materia, l'essere divisibile, il demiurgo, il mondo delle idee), le quali avevano un'esistenza reale e non solo logica ${ }^{14}$.

Per Plutarco tra le entità che precedono la nascita del cosmo c'è anche l'anima irrazionale, alla quale Platone nel passo sulla generazione dell'anima del mondo si riferì con la formula oủoía

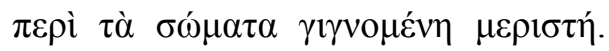
Lo stato dell'universo prima della generazione è costituito da una massa corporea dotata di un movimento incessante e caotico:

Infatti, ciò che esisteva prima della generazione del cosmo era

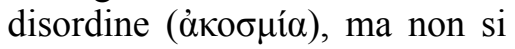
trattava di un disordine privo di corpo, di movimento e di anima, bensì di un disordine dotato di una corporeità senza forma e coesione e di una motricità scomposta e irrazionale (An. procr. 5. 1014B) ${ }^{15}$.

Il ragionamento che ha condotto Plutarco a ipotizzare l'esistenza di un' anima precosmica viene ricostruito da Proclo in una celebre testimonianza nella quale al filosofo di Cheronea viene affiancato anche Attico, un medioplatonico le cui posizioni furono molto vicine a quelle di Plutarco:

Plutarco e Attico si attaccano con tenacia a queste parole (scil.:

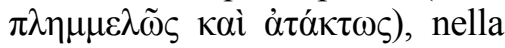
convinzione che esse testimonierebbero in favore della loro tesi relativa alla generazione del cosmo a partire da un tempo. Inoltre essi sostengono che prima della generazione esisteva non solo la materia priva di ordine ma esisteva anche l'anima produttrice di

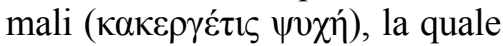
muoveva questo caos. Da dove infatti proveniva il movimento se non dall'anima? E se il movimento era disordinato, esso proveniva da un'anima disordinata (Procl. In Tim. 1. 381,26 ss. $=$ Attic. fr. 23 des Places).

Proclo sintetizza dunque la strategia argomentativa di Plutarco (e di Attico) nei seguenti termini: se Platone sostiene che lo stato dell'universo prima della generazione del cosmo era caratterizzato dalla presenza di movimento, sia pure disordinato e privo di regola, e se l'anima è per lui principio e causa di ogni movimento, allora, per spiegare la presenza del movimento nella fase precosmica, bisogna postulare l'esistenza di un'anima, evidentemente precosmica. Si tratta di un'anima, priva di intelligenza (ővov $\varsigma$ ) e irrazionale, la quale è responsabile del movimento caotico che sconvolge il sostrato ma-

$14 \mathrm{Si}$ veda quanto scrive in proposito Opsomer, 2004, pp. 146-47.

15 Cfr. in proposito il commento ad locum in FerRARI 2002, pp. 243-44. 
teriale prima dell'intervento del demiurgo.

L'ipotesi di quest'anima precosmica irrazionale non ha solo la funzione di spiegare la presenza del movimento nella fase anteriore alla generazione dell'universo; essa consente a Plutarco anche di risolvere il delicato problema relativo all'origine del male, cioè, per un platonico, del disordine. In base a una celebre indicazione formulata da Platone alla fine del II libro della Repubblica, dio non può essere responsabile del male; tuttavia, a differenza di quanto pensavano gli stoici, il male non può derivare dal non essere, risultando di

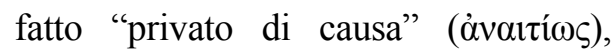
e neppure, come forse ritenevano alcuni platonici, dalla materia, perché

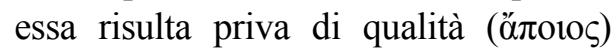
e indifferente (ådiópopos) dal punto di vista assiologico. L'accusa che l'aristotelico Eudemo mosse a Platone di avere attribuito alla materia, che nel Timeo viene chiamata "madre e nutrice", la causa dei mali risulta dunque del tutto infondata, dal momento che per Platone la causa del mali non è la materia, che è indifferente, bensì "la forza che mette in movimento la materia", vale a dire l'anima (An. procr. 7. 1015D-E).
Materia priva di forma e anima irrazionale, dunque, si coappartengono

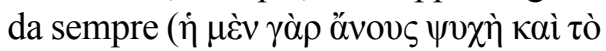

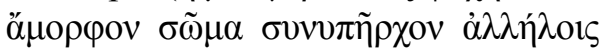
àeí: Plat. quaest. 4. 1003A), perché sono eterne, come eterno è anche il demiurgo. Esse si possono distinguere solo dal punto di vista logico non temporale, appunto perché esistono da sempre e non c'è mai stato uno stadio in cui la materia precosmica è esistita indipendentemente dall'anima ${ }^{16}$. Tuttavia non sono identiche, perché la materia è indifferente e priva di qualità, mentre l'anima irrazionale è causa e principio del movimento disordinato, e dunque, per un platonico, del male.

\section{$\mathrm{V}$}

A questo punto viene naturale chiedersi come Plutarco fosse arrivato a formulare una simile concezione, che sarebbe davvero difficile rintracciare nei dialoghi platonici. In realtà per riuscire ad attribuire a Platone la dottrina dell'anima precosmica Plutarco si servì di un metodo di ermeneutica testuale abbastanza diffuso tra gli interpreti medioplatonici dei dialoghi. Si tratta della norma ermeneutica che invita a chiarire "Platone a partire da Platone"

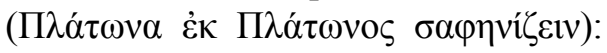

16 Sulla coappartenenza precosmica di anima e materia cfr. THÉVENAZ, 1938, pp. 108-14 e FERRARI, 1995, pp. 86-90. Sull'impossibilità di risalire a una fase precosmica embrionale nella quale la materia si presentasse inerte, ossia non compenetrata dall'anima precosmica, cfr. RESCigno, 1997, pp. 63-6. Osservazioni intelligenti sul rapporto tra materia e anima nella fase precosmica si trovano anche nel vecchio articolo di DeL RE, 1950, pp. 38-44. 
di fronte alle difficoltà che si incontrano dinnanzi a un passo particolarmente oscuro, questa norma suggerisce di servirsi di un altro passo platonico, nel quale la medesima concezione viene formulata in modo più chiaro e diretto. Naturalmente 1'uso di questo principio di esegesi testuale presuppone l'assunzione dell'idea che la filosofia platonica sia coerente e unitaria, sia cioè sistematica. Tutto ciò trovò espressione in un celebre principio che si trova formulato in Stobeo e che forse risale, attraverso Ario Didimo, a Eudoro di

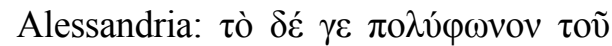

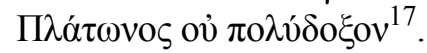

Plutarco si servì del principio sistematico e della norma che invitava a "chiarire Platone a partire da Platone" nel momento in cui si propose di interpretare il significato

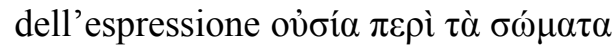

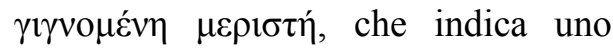
degli ingredienti della composizione dell'anima cosmica. Egli si rivolse a un celebre e misterioso passo del X libro delle Leggi, dove Platone alludeva all'esistenza di almeno due anime, "quella che produce il bene e quella che è capace di produrre il contrario" (Leg. 10. 896E). Questa seconda anima rappresenta per Plutarco l'anima precosmica che nel Timeo entra nella composizione dell'anima del mondo sotto forma di "essere divisibile nei corpi". Presentando questa anima irrazionale, Plutarco avverte in maniera acuta l'esigenza di distinguerla dalla materia, come dimostra questa importante affermazione:

È vero che Platone chiama madre e nutrice la materia, ma ciò che egli chiama causa del male è il movimento che muove la materia, il quale è divisibile nei corpi, disordinato, irrazionale e non inanimato. Si tratta di ciò che nelle Leggi, come si è detto, egli ha chiamato 'anima opposta e controbilanciante il principio che produce il bene'. L'anima è infatti causa e principio di movimento, mentre l'intelletto lo è dell'ordine e della consonanza che si trovano nel movimento. Perché dio non svegliò la materia intorpidita, ma ne arrestò lo sconvolgimento prodotto dalla causa priva di intelligenza (An. procr. 7. 1015D-E).

Ma il passo delle Leggi in cui Platone accenna a un' anima produttrice di effetti negativi non è l'unico luogo dei dialoghi in cui, secondo Plutarco, l'esistenza dell'anima precosmica viene evocata. Si tratta solo del riferimento più esplicito e diretto. Il fatto è che gli altri accenni all'anima precosmica irrazionale non risultano im-

17 Stob. Anth. 2.49,25-50,1. Sui principi esegetici di cui fecero uso gli interpreti medioplatonici e in particolare sulla norma del Platonem ex Platone e sull'assunto della coerenza e sistematicità del pensiero platonico rinvio a FERRARI, 2001, pp. 533-43 e più recentemente FERRARI, 2010, pp. 59-64. 
mediatamente riconoscibili, perché Platone usa una terminologia di volta in volta diversa. Ma quando, nel Timeo (48A), egli parla della "necessità" (àvó $\gamma \kappa \eta)$, oppure, nel Filebo (24A-25A

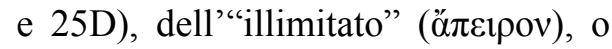
ancora, nel Politico (272E; 273B), del "desiderio connaturato" ( $\sigma 0 ́ \mu \varphi v \tau o \varsigma$ $\dot{\varepsilon} \pi(\theta v \mu i ́ \alpha)$, egli si riferisce sempre alla medesima entità, cioè all'anima precosmica irrazionale, principio del movimento caotico e disordinato del corpo del mondo prima della generazione dell'universo (An. procr. 6. 1014D-1015B) ${ }^{18}$.

Per chi, come Plutarco, si propone di dimostrare che l'anima in sé fa parte delle entità che preesistono alla generazione del cosmo, c'è un passo del Timeo che merita la massima attenzione, perché in esso Platone sembra descrivere la condizione del tutto prima della nascita dell'universo. Si tratta del celebre riassunto contenuto in $52 \mathrm{D}$, in cui vengono esplicitamente indicate le tre entità che preesistono al cosmo

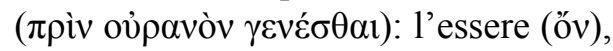

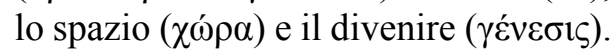

Plutarco riporta e commenta questo passo nel capitolo 24 del De procreatione, e si dimostra perfetta- mente consapevole dell'importanza che esso riveste per la sua interpretazione. Egli identifica in maniera automatica l'essere con il mondo delle idee (ed eventualmente, come vedremo, con la divinità), e lo spazio con la materia. Molto più complessa, e tuttavia inevitabile, si profila invece l'identificazione della generazione ( $\gamma \varepsilon \dot{v} v \sigma \sigma \varsigma)$ con l'anima precosmica $^{19}$. Ma egli è perfettamente consapevole che le basi stesse della sua interpretazione verrebbero scosse in maniera irrimediabile se l'anima non venisse menzionata da Platone proprio all'interno della descrizione dello stato precosmico dell'universo. E dunque non si fa alcun scrupolo a stabilire l'equivalenza tra la generazione e l'anima precosmica irrazionale:

Egli [Platone] chiama spazio la materia, intendendo che è sede e ricettacolo; chiama essere l'intelligibile, mentre la generazione, quando il cosmo non era ancora venuto all'essere, non è altro che la sostanza immersa in mutamenti e movimenti, collocata in mezzo tra ciò che imprime e ciò che viene impresso, e che trasmette quaggiù le immagini di lassù. Per questa ragio-

18 Un'eccellente analisi dei passi invocati da Plutarco a sostegno dell'attribuzione a Platone della concezione dell'anima precosmica irrazionale si trova in OPSOMER, 2004, pp. 14852; cfr. anche BaLtes, 2000, p. 250.

19 Un flebile sostegno in favore dell'identificazione della $\gamma \varepsilon ́ v \varepsilon \sigma 1 \varsigma$ con l'anima precosmica potè venire a Plutarco dal riferimento a Leg. 10. 895A, dove l'anima viene appunto

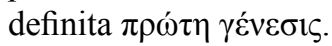


ne è stata definita 'divisibile'...

(An. procr. 24. 1024C) $)^{20}$.

Si direbbe che Plutarco presenti qui una nuova e originale teoria dei principi, sostanzialmente sconosciuta agli altri autori medioplatonici. Essa tuttavia non va considerata alternativa ad altre diffuse negli autori di questo periodo e in Plutarco stesso, perché si riferisce unicamente allo stato del mondo prima dell'intervento cosmopoietico del demiurgo.

Non c'è dubbio che Plutarco fraintese il senso delle parole platoniche, e in particolare fraintese il significato

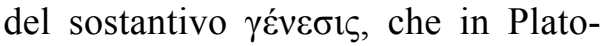
ne è evidentemente sinonimo di $\gamma / \gamma$ vó $\varepsilon_{\varepsilon v o v}$ e indica semplicemente il cosmo sensibile, che si trova nel mezzo dell'essere e dello spazio (materia) in quanto viene da essi generato. Viceversa Plutarco intende l'intermedietà della $\gamma \varepsilon ́ v \varepsilon \sigma 1 \varsigma$ come se dovesse venire riferita all'anima precosmica, che è intermedia in quanto consente alla materia di ricevere l'intelligibile, o meglio le tracce

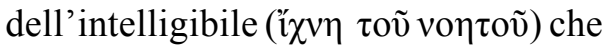
agiscono nella fase precosmica (cfr.
An. procr. 9. 1016D-1017A). Se non fosse permeata dall'anima, la materia non potrebbe venire "impressa", non potrebbe cioè ricevere le tracce provenienti dall'intelligibile, il quale risulta, stando alle stesse affermazioni platoniche, attivo in una qualche misura anche nello stadio precosmico (Tim. 53A-B). Il ricorso all'ipotesi dell'anima precosmica, dunque, non consente a Plutarco solamente di spiegare la presenza del movimento del sostrato prima della cosmogenesi, ma gli permette anche di dare conto dei passi in cui Platone sembra accennare alla circostanza che l'intelligibile agisce nel sostrato anche prima della generazione dell'universo ${ }^{21}$.

Come si vede, l'ipotesi dell'anima precosmica irrazionale appare strettamente collegata all'esigenza di fornire un'interpretazione letterale del Timeo, in grado di inserire in un quadro unitario e coerente tutte le affermazioni contenute nel testo. Da questo punto di vista Plutarco si profila davvero come il campione dell'esegesi letterale di Platone.

20 Sull'identificazione operata da Plutarco tra la generazione di Tim. 52D e l'anima precosmica si trovano importanti osservazioni in OPSOMER, 2004, pp. 152-153.

21 Per un quadro delle questioni collegate alla concezione plutarchea della generazione precosmica, ossia di una forma embrionale di ordinamento attiva anche prima dell'intervento cosmopoietico del demiurgo, rinvio a FERRARI, 1995, pp. 90-8, FERRARI, 1996, passim, e Rescigno, 1997. Si veda anche Baltes, 2000, p. 249, il quale sottolinea come l'anima precosmica sia intermedia tra gli intelligibili e la materia precosmica in quanto riproduce le immagini attraverso le quali la realtà intelligibile imprime il caos precosmico. 


\section{VI}

Proprio l'impostazione letteralistica dell'esegesi di Plutarco sembrerebbe subire uno scacco dal confronto di alcuni passi del corpus, nei quali Platone parla dell'anima in termini apparentemente contraddittori, definendola sia "ingenerata" ( $\mathrm{ad}$ es. in Phaedr. 245C-246A), sia "generata" dal demiurgo (in Tim. 37A). In realtà, secondo Plutarco una corretta esegesi di questi passi, effettuata alla luce della concezione dell'anima precosmica, consente addirittura di pervenire a un'ulteriore conferma della correttezza della sua interpretazione. Partendo da essa, infatti, è possibile risolvere l'apparente incongruenza

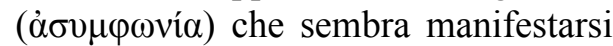
in ciò che Platone afferma a proposito dell'anima, definita appunto "ingene-

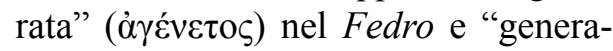

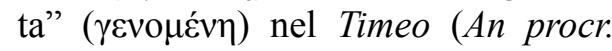
8. 1015F-1016A). Si tenga presente che l'accusa di incoerenza e contraddittorietà (lat.: inconstantia) era una di quelle più frequentemente mosse a Platone dai suoi avversari, e in particolare da stoici ed epicurei ${ }^{22}$.

Servendosi di una raffinata strategia ermeneutica Plutarco dimostra che l'ipotesi dell'anima precosmica con- sente di difendere Platone da questa accusa. Infatti le affermazioni contenute nel Fedro e nel Timeo non si riferiscono alla stessa anima, ma a due anime differenti: quella del $\mathrm{Fe}$ dro riguarda l'anima precosmica, che è infatti eterna e ingenerata, mentre quella del Timeo si riferisce all'anima del mondo, che è generata dal demiurgo. La prima anima muove il corpo precosmico, la seconda muove razionalmente il corpo del mondo:

Dichiara infatti che è ingenerata l'anima che muove tutte le cose in modo discorde e disordinato prima della generazione del cosmo, mentre è venuta all'essere ed è perciò generata quell'anima che dio ha collocato come guida dell'universo, dopo averla resa intelligente e ordinata a partire da questa [l'anima precosmica] e dall'essere stabile e migliore (An. procr. 9. 1016C) ${ }^{23}$.

Naturalmente si possono rivolgere numerose obiezioni a una simile interpretazione. Un commentatore dei nostri giorni difficilmente potrebbe sostenere che essa restituisca in modo corretto il punto di vista di Platone, soprattutto perché non sarebbe affatto disposto ad accettare l'identificazione plutarchea tra l'anima del Fedro e

22 Cfr. in proposito Ferrari, 2001, pp. 538-43 e Ferrari, 2010, pp. 56-9.

23 In realtà, come ha osservato con finezza OPSOMER, 2004, p. 153, l'anima precosmica e l'anima del mondo non sono numericamente diverse, non sono cioè due anime distinte, ma la medesima anima in due fasi differenti, cioè prima e dopo l'ordinamento del demiurgo. 
l'anima precosmica irrazionale. Ma non c'è dubbio che l'esegesi di Plutarco costituisca una maniera sensata ed elegante di difendere Platone dall'accusa di inconstantia.

Questo è tanto più vero ove si consideri che Plutarco si servì implicitamente di una celebre norma ermeneutica stabilita da Aristotele nel capitolo 25 della Poetica (1061b1719), dove egli invitava, di fronte ai casi in cui un autore sembra fare affermazioni contraddittorie, a stabilire se tali affermazioni si riferiscono effettivamente alla medesima realtà. Plutarco si è proposto di dimostrare che le affermazioni di Platone intorno all'anima, definita "ingenerata" nel Fedro e "generata" nel Timeo, non si riferiscono alla stessa entità ma a due realtà diverse, cioè all'anima precosmica e all'anima del mondo, o meglio, si riferiscono alla medesima anima in due stadi diversi, quello precosmico e quello cosmico ${ }^{24}$.

\section{VII}

Leggendo la prima parte del trattato sulla generazione dell'anima nel Timeo si è inevitabilmente portati a constatare come la maggior parte degli sforzi esegetici dell'autore sia consacrata a dimostrare che l'essere divisibile che diviene nei corpi vada identificato con l'anima precosmica, cioè con l'essenza stessa della psichicità. In effetti Plutarco non dedica uguale attenzione e impegno a chiarire che cosa sia l'essere indivisibile che permane sempre identico, forse perché l'individuazione di questa entità si presentava per un platonico meno problematica.

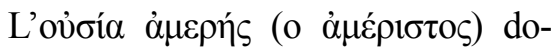
vrebbe venire identificata d'emblée con la realtà intelligibile, ossia con il mondo delle idee, come in effetti fecero, in forma più o meno esplicita, molti platonici prima di Plutarco. Quest'ultimo tuttavia non assunse una simile posizione, o almeno non l' assunse in maniera univoca. Indotto probabilmente da ragioni di simmetria teorica ${ }^{25}$, Plutarco sembra infatti identificare la sostanza indivisibile con l'intelletto (voṽs) e non con le idee. Per pervenire a una simile conclusione egli potrebbe avere ragionato pressappoco in questi termini: se l'essere divisibile è un'anima irrazionale, allora la sua controparte, cioè l'essere indivisibile e sempre identico, dovrà essere un intelletto, ossia un'entità alla quale sarebbe naturale per un platonico assegnare la causa dell'ordine e della razionalità.

E questa sembra in effetti la posizione che Plutarco assunse, per lo più in forma implicita, lungo l'intera prima parte del trattato (cfr., per es., 6. 1014E;

24 Si veda la nota precedente. Sulla strategia esegetica di Plutarco volta a eliminare le presunte incongruenze di Platone cfr. FERRARI, 2002, pp. 22-5 e 260 ss.

25 Così ritiene, probabilmente non senza fondamento, CHERNISS, 1976, pp. 141-43. 
23. 1024A; 24. 1024D), dove l'intelletto sembra entrare direttamente nell'anima irrazionale, trasformandola così in anima del mondo. Tuttavia in alcuni passi, per esempio in 9. 1016C-D e soprattutto in 22. 1023C-D, egli sembra distinguere in maniera molto netta l'intelletto demiurgico dall'essere indivisibile (cioè dal mondo delle idee), attribuendo al primo una funzione demiurgico-efficiente, e al secondo un ruolo paradigmatico ${ }^{26}$. Probabilmente non si tratta di vere e proprie incoerenze, ma del fatto che Plutarco non fu veramente interessato a operare distinzioni chiare e definitive all'interno della sfera intelligibile, preferendo assumere un atteggiamento teorico piuttosto flessibile. Ai suoi occhi di pla-

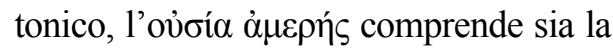
funzione paradigmatica sia quella efficiente e demiurgica; e del resto, come egli afferma in un celebre passo della

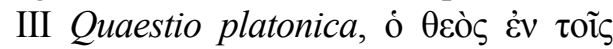

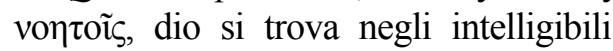
(1002B).

La generazione dell'anima cosmica è concepita da Plutarco come l'esito dell'introduzione da parte del demiurgo di intelletto, razionalità e armonia nel sostrato psichico irrazionale. Il complesso di questi caratteri viene talora indicato con il nome collettivo di

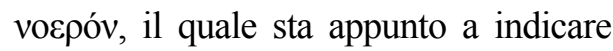

la sfera della razionalità, il cui ingresso trasforma il sostrato psichico precosmico in anima del mondo. Plutarco afferma che dio ha trasformato il sostrato psichico irrazionale in anima del mondo

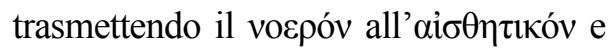

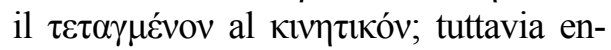
trambi questi aspetti non provengono dal di fuori, ma dio li ha ricavati da se stesso

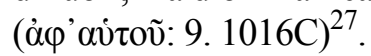

La medesima tesi sembra emergere in un celebre e misterioso passo delle Quaestiones platonicae, dove Plutarco ricorre a una descrizione metonimica e preposizionale per esporre il rapporto tra dio el' anima del mondo. Egli afferma che dio è $\pi \alpha \tau$ ๆ́ dell'anima in quanto essa non rappresenta solo un prodotto

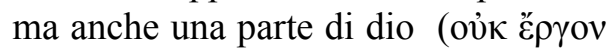

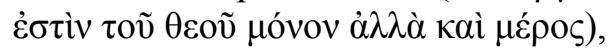
e aggiunge, ricorrendo al linguaggio metonimico molto diffuso nelle scuole,

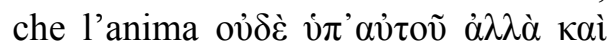

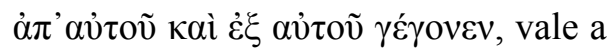
dire che essa non è tanto (e solo) nata in virtù dell'intervento di dio come causa efficiente, ma deriva da dio ed è costituita di una parte (il vocpóv?) di dio (Plat. quaest. 2. 1001C) $)^{28}$.

Tutto ciò sembra avvalorare l'ipotesi che Plutarco si rappresenti la genesi dell' anima cosmica comel'introduzione da parte dell'intelletto demiurgico di

26 Cfr. in proposito Ferrari, 1995, pp. 245-46 e Ferrari, 2002, pp. 45-8.

27

28 Sulla parte conclusiva della II quaestio platonica devo rinviare a FERRARI, 2006, pp. 55-7 e soprattutto a BALTES, 2008, pp. 577-80. 
principi noetici e razionali all'interno dell'anima precosmica, e che questi principi noetico-razionali siano parti della sostanza demiurgico-divina.

\section{VIII}

Per Plutarco, dunque, la generazione dell'anima cosmica rappresenta un evento reale (non una metafora) che si produce nel momento in cui dio entra in qualche forma, ossia attraverso il

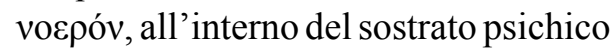
precosmico indeterminato. Le due componenti fondamentali dell'anima del mondo sono perciò l'anima precosmica e l'intelletto demiurgico. Ma essi non sono i soli ingredienti, dal momento che Platone, come abbiamo visto, menziona altri due principi che entrano nella composizione finale dell' anima: l'identico e il diverso.

Lo schema compositivo che Plutarco ricava dal passo del Timeo è notevolmente semplificato rispetto all' originale platonico. Plutarco arriva a operare questa semplificazione anche per mezzo di una serie di interventi testuali, che egli introduce personalmente o recepisce dalla tradizione, e che sono certamente funzionali a supportare la sua personale esegesi del testo del Timeo $^{29}$.

Nel capitolo 25 del trattato sulla generazione dell'anima emerge con evidenza la semplificazione della struttura compositiva dell'anima cos- mica operata da Plutarco. Egli invita infatti a considerare la maniera in cui il demiurgo ha attuato la fusione degli elementi che formano il corpo del

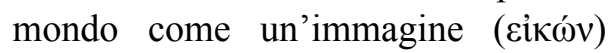
del modo in cui egli ha armonizzato gli ingredienti dell'anima. Nel caso del corpo, due dei quattro elementi fondamentali, e per la precisione fuoco e terra, risultavano non mescolabili direttamente in quanto "assolutamente incompatibili e inconciliabili”. Di fronte all'impossibilità di mescolare direttamente il fuoco e la terra, il demiurgo ha collocato in mezzo ad essi l'acqua (dalla parte della terra) e l'aria (dalla parte del fuoco), li ha mescolati tra loro e poi ha mescolato il prodotto di questa fusione con i due estremi ( $A n$. procr. 25. 1025A).

Secondo Plutarco dio ha proceduto alla stessa maniera nel caso dell' anima: i due estremi inconciliabili sono l'identico e il diverso, i quali non possono venire mescolati direttamente; per questo dio ha collocato in mezzo l'essere indivisibile e l'essere divisibile, li ha mescolati tra loro e poi ha operato la mescolanza finale:

Anche nel nostro caso riunì insieme l'identico e il diverso, che sono potenze ed estremi che si controbilanciano, ma non direttamente tra loro, bensì dispose in funzione di intermediari altre

29 Cfr. in proposito Cherniss, 1976, pp. 158-61, Hershbell, 1987, p. 240, FerRari, 1999, p. 332 ss. e OpsOMER, 2004, pp. 139-42 e 159-61. 
sostanze, e cioè quella indivisibile davanti all'identico e quella divisibile davanti al diverso, ciascuna delle quali si adatta a uno degli estremi, quindi con un'ulteriore mescolanza li unì con quelle sostanze che erano già state mescolate tra loro $(25.1025 \mathrm{~B})^{30}$.

Come si vede, lo schema risulta radicalmente modificato e significativamente semplificato rispetto a quello ricavabile dal Timeo (gli ingredienti sono ridotti a quattro, mentre erano sei nel testo platonico). In Plutarco identico e diverso entrano direttamente nella composizione finale dell'anima, e non sembrano ammettere una mescolanza intermedia come in Platone. Plutarco non accenna a un identico mediano e a un diverso mediano, ma prende in considerazione queste due entità in modo assoluto, non chiarendo se si tratti di sostanze indivisibili o divisibili. In realtà il loro status ontologico non può non risultare problematico ove si presti attenzione alla circostanza che essi occupano nello schema di Plutarco i poli estremi, trovandosi dunque al di "sopra" dell'essere indivisibile (l'identico) e al di "sotto" dell'essere divisibile nei corpi (il diverso).

In realtà identico e diverso vengono implicitamente trattati da Plutarco non come entità bensì come funzioni, sebbene egli non approfondisca affatto questo motivo, che meriterebbe probabilmente di venire indagato dalla critica in maniera più analitica di quanto sia possibile fare in questa sede. Non c'è dubbio, comunque, che Plutarco attribuisca a identico e diverso un ruolo di natura epistemologica e cosmologica (ad es. l'identico, forse riconducibile al principio dell'uno, è responsabile del movimento della sfera delle stelle fisse, mentre il diverso, espressione della diade, causa il movimento errante dei pianeti: cfr. An. procr. 28. 1026E-1027A), lasciando sostanzialmente ai margini la discussione della loro eventuale funzione ontologica ${ }^{31}$.

Merita infine un rapido accenno anche il tema delle anime individuali, le quali già nel Timeo dipendevano dall'anima cosmica. Plutarco cerca di chiarire il senso di questa dipendenza, spiegando che la composizione dell' anima cosmica si rispecchia anche nella struttura di quella individuale, che è anch'essa formata da un elemento razionale e monadico, cioè indivisibile, e da un elemento passionale e diadico, cioè divisibile (An. procr. 26. 1025D). Dal momento che l'anima individuale è "parte e copia" dell'anima del mon-

30 Sullo schema compositivo che Plutarco applica sia all'anima che al corpo del mondo cfr. SCHOPPE, 1994, pp. 109-15 e OPSOMER, 1994, pp. 39-40.

31 Giusti rilievi si leggono in Opsomer, 2004, pp. 144-46. Si veda anche FERRARI, 2002, pp. 48 52. Sul ruolo di identico e diverso vale la pena consultare anche THÉvenAz, 1938, pp. 80-4. 
do (Virt. mor. 441F), essa presenta i medesimi ingredienti di quest'ultima, con la differenza che nell'anima individuale "si manifesta in misura maggiore l'aspetto diadico, mentre l'aspetto della parte semplice e monadica risulta sommerso in una maggiore oscurità" (An. procr. 1025D).

In questo modo Plutarco si propone di dimostrare che la natura composita e duale dell'anima degli individui, nella quale l'elemento irrazionale è connaturato (dipendendo dall'anima del mondo, e in particolare dal fatto che uno degli ingredienti fondamentali di essa è rappresentato dall'anima precosmica irrazionale), rinvia in ultima analisi alla struttura dell'anima cosmica. Del resto la divisione della parte irrazionale dell'anima individuale

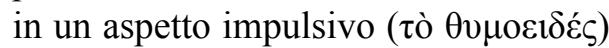
e in uno desiderativo ( risulta secondaria rispetto alla bipartizione fondamentale tra ragione $\mathrm{e}$ passione ${ }^{32}$. Sbagliano dunque gli stoici a ritenere che l'anima sia una entità monolitica e unitaria, e che le passioni siano giudizi errati. Le passioni sono invece manifestazioni ineliminabili di una parte dell'anima individuale, che dipende dalla composizione dell' anima del mondo, di cui le anime individuali sono copie. In questo modo Plutarco tenta di fornire un fondamento ontologico e cosmologico alla sua concezione etica della metriopatheia, sviluppata in maniera analitica nello scritto De virtute morali.

\section{IX}

In conclusione di queste pagine si possono proporre alcune riflessioni di carattere generale sulla natura dello trattato plutarcheo dedicato alla generazione dell'anima cosmica e sulle linee fondamentali dell'interpretazione che in esso viene sviluppata.

(1) Lo scritto rappresenta senza dubbio un tentativo coraggioso e non privo di consistenza filosofica di affrontare l'esegesi di uno dei passi più controversi del corpus platonico. Il De animae procreatione si inserisce all'interno della tradizione dei cosiddetti Spezialkommentare, commentari specialistici, attraverso i quali i platonici dei primi secoli dell'epoca imperiale affrontavano l'esegesi dei dialoghi del maestro. (2) In aperta polemica con la tendenza ormai dominante nel platonismo della sua età, Plutarco fornisce un'esegesi letterale del Timeo e in particolare della cosmogenesi in esso descritta. Insieme ad Attico egli è destinato a restare, almeno tra gli autori pagani, il più agguerrito partigiano dell'interpretazione "temporale" del Timeo. (3) La generazione del cosmo così come quella della sua anima sono dunque eventi reali. Essi vengono

32 Sulla dipendenza della struttura dell'anima individuale dalla composizione dell'anima cosmica si veda OPSOMER, 1994, pp. 42-4. 
attribuiti da Plutarco, conformemente alla sua esegesi letterale del Timeo, a un intelletto demiurgico, la cui azione non consiste nella generazione ex nihilo, ma nell'ordinamento di un sostrato indeterminato, il quale transita da uno stato precosmico di disordine a una condizione cosmica caratterizzata da ordine ed armonia. (4) Prima della generazione dell'universo esisteva un sostrato psico-somatico caratterizzato da corporeità informe e da movimento caotico. Ciò significa che il movimento disordinato che attraversa la materia indeterminata è causato da un'anima precosmica irrazionale, in ottemperanza al principio "platonico" secondo il quale l' anima è causa di ogni movimento. (5) L'esame degli ingredienti che entrano nella composizione dell'anima cosmica consente a Plutarco di individuare quest'anima precosmica sotto

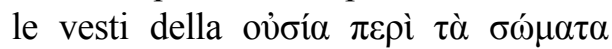
$\gamma \iota \gamma v 0 \mu \varepsilon ́ v \eta \eta$ perché si divide nei corpi e dunque non può essere un corpo, ma è un'entità in grado di compenetrare il corpo. (6) Una simile concezione fa di Plutarco il primo platonico a concepire esplicitamente l'anima non come un principio di ordine e di armonia, ma come la causa di una motricità assoluta e irrazionale: l'anima

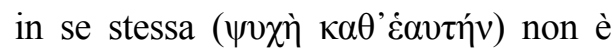
un principio razionale, ma costituisce la dimensione della pura irrazionalità, ossia del disordine. (7) L'ipotesi dell' esistenza di un'anima precosmica consente poi a Plutarco di trovare una spiegazione della presenza del male nel cosmo, evitando di incorrerere nelle aporie in cui si erano invischiate le dottrine degli stoici e degli epicurei. (8) Agli occhi di Plutarco l'ipotesi che l'anima del mondo si generi a partire da un sostrato precosmico che è già di natura psichica permette di spiegare la presenza in essa di caratteristiche e funzioni riconducibili alla dimensione psichica, le quali sarebbero incomprensibili se si accettassero le interpretazioni geometrico-matematiche o materialistiche avanzate da $\mathrm{al} \cdot \mathrm{cu} \cdot \mathrm{ni}$ autorevoli platonici dei secoli precedenti. (9) Infine Plutarco ritiene che un'esegesi come quella da lui avanzata stabilisca un ponte tra la dimensione cosmica e quella individuale, dal momento che l'anima del singolo, in quanto "parte e copia" di quella del mondo, presenta $i$ medesimi ingredienti di quest'ultima.

Molte delle tesi di Plutarco non potranno soddisfare il commentatore dei nostri giorni, al quale le interpretazioni discusse in queste pagine sembreranno probabilmente il frutto di assunzioni arbitrarie, come del resto aveva lamentato, forse con un eccesso di spirito polemico, il grande Harold Cherniss. Non c'è dubbio, tuttavia, che l'autore del De animae procreatione dimostri di essere un lettore di Platone non privo di acutezza e profondità, e che il suo sforzo di fornire un'immagine coerente e unitaria del pensiero del maestro meriti tutto il nostro rispetto. 


\section{Bibliografia}

BALtes, M.,

- Die Weltentstehung des Platonischen Timaios nach den antiken Interpreten, Bd. 1, Leiden, 1976.

- "La dottrina dell'anima in Plutarco", Elenchos, 21 (2000) 245-70.

- Der Platonismus in der Antike, Bd. 7.1: Theologia Platonica, Stuttgart-Bad Cannstatt, 2008.

BRISSON, L.,

- Le Même et l'Autre dans la structure ontologique $d u$ Timée de Platon. Un commentaire systématique du Timée de Platon, Sankt Augustin, 1994.

Cherniss, H.,

- Plutarch's On the Generation of the Soul in the 'Timaeus', in Plutarch's Moralia, XIII, Cambridge Mass., 1976.

Del Re, R.,

- "Il pensiero metafisico di Plutarco: Dio, la natura, il male", Studi Italiani di Filologia Classica, 24 (1950) 33-64.

Deuse, W.,

- Untersuchungen zur mittelplatonischen und neuplatonischen Seelenlehre, Wiesbaden, 1983.

FERRARI, F.,

- Dio, idee e materia. La struttura del cosmo in Plutarco di Cheronea, Napoli, 1995.

- "La generazione precosmica e la struttura della materia in Plutarco", Museum Helveticum, 53 (1996) 44-55.

- "Platone, Tim. 35A1-6 in Plutarco, An. Procr. 1012B-C: citazione ed esegesi", Rheinisches Museum für Philologie, 142 (1999) 326-39.

- Plutarco, La generazione dell'anima nel 'Timeo', Introduzione, testo critico, traduzione e commento, a cura di F. Ferrari e L. Baldi, Napoli, 2002 (Corpus Plutarchi Moralium, 37).

- "Struttura e funzione dell'esegesi tes- tuale nel medioplatonismo: il caso del Timeo", Athenaeum, 89 (2001) 525-74.

- "Poietes kai pater: esegesi medioplatoniche di Timeo, 28c3", in G. De Gregorio - S. Medaglia (a cura), Tradizione, ecdotica, esegesi, Miscellanea di studi, Napoli, 2006, 43-58.

- "Esegesi, commento e sistema nel medioplatonismo", in A. NeschKeHentschKe (Hrsg.), Argumenta in dialogos Platonis, Teil 1: Platoninterpretation und ihre Hermeneutik von der Antike bis zum Beginn des 19. Jahrhunderts, Basel, 2010, 51-76.

Hershbell, J.P.,

- "Plutarch's De animae procreatione in Timaeo: An Analysis of Structure and Content", ANRW II 36.1 (1987) 234-47.

Opsomer, J.,

- "L'Âme du monde et l'âme de l'homme chez Plutarque", in M. Garcia ValdÉs (ed.), Estudios sobre Plutarco: Ideas religiosas, Madrid, 1994, 33-49.

- "Plutarch's De animae procreatione in Timaeo: Manipulation or Search for Consistency?", in P. AdAmson - H. Baltussen - M.W.F. Stone (eds.), Philosophy, Science and Exegesis in Greek, Arabic and Latin Commentaries, vol. 1, London 2004, 137-62.

Rescigno, A.,

- "Desiderare componi a deo. Attico, Plutarco, Numenio sulla materia prima della creazione", Koinonia, 21 (1997) 39-81.

Schoppe, C.,

- Plutarchs Interpretation der Ideenlehre Platons, Münster-Hamburg, 1994.

ThÉvenAz, P.,

- L'âme du monde, le devenir et la matière chez Plutarque. Avec une traduction du traité De la Genèse de l'Âme dans le Timée ( $1^{\text {re }}$ partie), Neuchatel, 1938. 